\title{
Widespread mechanical pain hypersensitivity in patients with chronic migraine and temporomandibular disorders: relationship and correlation between psychological and sensorimotor variables
}

\author{
Miriam Garrigós-Pedrón ${ }^{a}$, Roy La Touche ${ }^{a, b}$, Pablo Navarro- Desentre ${ }^{c}$, \\ Manuel Gracia-Naya ${ }^{d}$ and Eva Segura-Ortí ${ }^{\prime}$

\begin{abstract}
${ }^{\text {a} D e p a r t a m e n t o ~ d e ~ F i s i o t e r a p i a, ~ M o t i o n ~ i n ~ B r a i n s ~ R e s e a r c h ~ G r o u p, ~ I n s t i t u t e ~ o f ~ N e u r o s c i e n c e ~ a n d ~ S c i e n c e s ~ o f ~ t h e ~ M o v e m e n t ~(I N C I M O V), ~}$

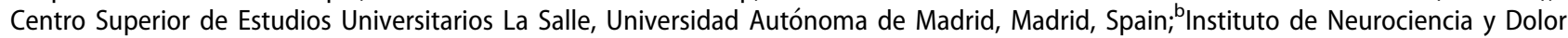
Fundación Atención Temprana, Zaragoza, Spain; ${ }^{\mathrm{d} D o c t o r}$ in Department of Neurology, Hospital Universitario Miguel Servet, Zaragoza, Spain; ${ }^{\mathrm{e} D e p a r t m e n t}$ of Physiotherapy, Universidad Cardenal Herrera-CEU, CEU Universities, Valencia, Spain
\end{abstract} \\ Craneofacial (INDCRAN), Instituto de Investigación Sanitaria del Hospital Universitario La Paz (IdiPAZ), Madrid, Spain;'Physiotherapist in
}

\begin{abstract}
Objective: This study aimed to assess mechanical hyperalgesia in the trigeminal and extra-trigeminal regions in patients with chronic migraine $(\mathrm{CM})$ and temporomandibular disorders (TMD) in comparison to asymptomatic subjects and to determine the association between sensorimotor variables and psychological and disability variables and evaluate the prediction of a sensorimotor variables though psychological and disability variables in patients with CM and TMD.

Material and methods: A total of 52 subjects with concomitant CM and TMD and 30 asymptomatic subjects were included in the study. The pressure pain threshold (PPT), maximal mouth opening (MMO) and a series of self-reported factors were compared.

Results: There were $52 \mathrm{CM}$ and TMD $(92.3 \%$ women and $7.7 \%$ men; age $=46.2 \pm 9.5)$ and 30 asymptomatic subjects $(80 \%$ women and $20 \%$ men; age $=47.4 \pm 10)$. Differences were found between patients with CM and TMD and asymptomatic participants $(p<.01)$ when comparing the PPTs in the trigeminal and extra-trigeminal regions. The PPT for the trigeminal region was predicted by depressive symptoms (variance of 18\%) as well as disability and craniofacial pain (variance of $20 \%$ ). The extratrigeminal region PPT was predicted by depressive symptoms (variance of $10 \%$ ), and pain-free MMO was predicted by disability and craniofacial pain (variance of 24\%).

Conclusions: This study suggests that patients with CM and TMD present with generalized mechanical hyperalgesia. In addition, an association between sensorimotor, psychological and disability variables was observed.
\end{abstract}

ARTICLE HISTORY

Received 27 February 2018

Revised 1 October 2018

Accepted 14 October 2018

\section{KEYWORDS}

Chronic migraine;

temporomandibular disorders; hyperalgesia; central sensitization; psycho-

logical factors

\section{Introduction}

Headaches, facial pain and temporomandibular disorders (TMD) of a chronic nature are highly prevalent in the general population [1]. These disorders represent a genuine health problem, and each disorder presents with cognitive, emotional and behavioral interactions [1-3]. These conditions can cause cutaneous allodynia and the sensitization of neurons in the trigeminocervical complex. Allodynia is the most obvious clinical sign of the existence of a central sensitization process [4] and is defined as a pain due to a stimulus that does not normally provoke pain $[5,6]$.

A chronic migraine $(\mathrm{CM})$ and TMD are associated with disability, have marked socioeconomic and personal impacts, and decrease the patient's health-related quality of life [7]. Most cases occur in females $[1,8,9]$. In addition to the physical symptomatology, psychological disorders are also present. When TMD causes pain located in the masticatory muscles, the temporomandibular joint (TMJ), and/or neighboring structures as its main symptom [9], patients suffer from elevated stress, depression and anxiety in comparison to control groups [3]. These patients also present with higher levels of catastrophization with regard to pain [2]. Patients with $\mathrm{CM}$ present with high levels of anxiety and depression [10].

As such, some degree of association between primary headaches (e.g. migraine) and TMD has been suggested [8]. Epidemiological studies have shown that TMD is commonly found in migraine patients. Indeed, these studies have shown that migraine prevalence increases in patients with TMD with respect to control groups $[8,11]$ and that the signs and symptoms of TMD are more common in patients with CM than in the general population [11].

A recent study has shown that the primary headache (e.g. migraine) and TMD are related and can, therefore, be considered to be different pathologies that act on each other, 
thereby perpetuating and worsening symptoms [11]. Evidence suggests that TMD may be a risk factor for the increased frequency of headaches observed in patients with CM and myofascial TMD is the most common type of TMD found in patients with CM [12].

The relationship between CM and TMD is complex. Consequently, some theories have attempted to explain this relationship from an anatomical and pathophysiological viewpoint through the trigeminal complex $[9,13,14]$.

A recent study has shown a weak correlation between pain intensity and pressure pain threshold (PPT) in patients with TMD [15]. As such, central sensitization factors and psychological variables have been suggested to be particularly relevant for explaining the pain experience in these patients [15]. Other study had shown that patients with TMD present with generalized hyperalgesia [16]. There is a lack of information on the prediction of sensorimotor variables as a PPT and maximal mouth opening (MMO) through psychological variables in patients with $\mathrm{CM}$ and TMD; thus, it is important to check if sensorimotor variables as a PPT and MMO could be predicted by psychological variables.

The primary aim of this study was to assess mechanical hyperalgesia in the trigeminal and extra-trigeminal region in a group of patients with CM and TMD and to compare the results to those obtained for a control group. The secondary aim was to determine the association between sensorimotor variables (PPT in the trigeminal and extra-trigeminal regions and $\mathrm{MMO}$ ) and psychological and disability variables and evaluate the prediction of sensorimotor variables though psychological and disability variables in patients with CM and TMD.

\section{Materials and methods}

\section{Study design}

This study used a cross-sectional design with a non-probabilistic sample. The authors compared two groups: one group of patients had CM and myofascial TMD, and one group was comprised of asymptomatic subjects who served as a control group (CG). After receiving detailed information about the study, all participants signed informed consent forms. All procedures used in this study were planned under the ethical guidelines of the Declaration of Helsinki and were approved by the local ethics committee and the ethics committee of the Hospital Universitario Miguel Servet (HUMS) in Zaragoza, Spain (approval number CP03/2015). This study follows the Strengthening the Reporting of Observational Studies in Epidemiology (STROBE) guidelines [17].

\section{Participant recruitment}

Patients were recruited at the Neurology Department of the HUMS. The inclusion criteria were as follows: (a) diagnosis of $\mathrm{CM}$, as assessed by a neurologist specializing in headaches and based on the criteria of the International Classification Headache Disorders-III (ICHD-III) of the International Headache Society (IHS) [18]; (b) patients aged between 21 and 65 years; and (c) diagnosis of myofascial TMD, as defined by the Research Diagnostic Criteria for TMD (RDC/TMD) [19]. All had a similar intake of routine medication consisting of continuous preventive treatment and abortive pharmacological treatment at the onset of migraine attacks prescribed by a headache specialist neurologist.

The exclusion criteria included the following factors: migraine crisis at the time of assessment, presence of other headache, another type of TMD, history of another chronic disease, history of neurological disease and/or dental problems and previous surgery or trauma to the upper body.

The healthy controls were volunteers recruited from the hospital and university environment. The subjects were between 21 and 65 years of age, and the exclusion criteria were a history of facial and/or head pain and/or dental problems, presence of acute or chronic musculoskeletal diseases, neurologic problems, systematic disease and/or use of pain medication and/or muscle relaxant medication.

\section{Procedure}

After consenting to participate in the study, the participants completed a battery of questionnaires on the day of the evaluation. These questionnaires collected data related to self-reported demographic data and pain-related variables. The socio-demographic questionnaire collected information about the following variables: age, gender, height, weight and duration of pain. Measures of pain were assessed using the Visual Analog Scale (VAS) [20]. Pain catastrophizing was assessed using the Pain Catastrophizing Scale (PCS) [21]. Pain related to disability in the craniomandibular and facial region was collected using the Craniofacial Pain and Disability Inventory (CF-PDI) [22]. The impact and severity of headache were quantified using the Spanish version of the Headache Impact Test-6 (HIT-6) [23]. The presence of signs and symptoms of depression and anxiety was assessed using the Spanish version of the Hospital Anxiety and Depression Scale (HADS) [24]. Once the patients had completed all self-reports, the researchers proceeded to evaluate the PPT and MMO without pain. PPT values were measured bilaterally over the masseter and temporalis muscles (trigeminal regions), based on a previously published protocol [25], and in the wrist (extra-trigeminal region). A blinded investigator performed the assessment of all measurements.

\section{Pain, psychological and disability self-reports}

The VAS was used to measure the intensity of pain perceived by the participants [20]. The VAS is a $10-\mathrm{cm}$ horizontal line anchored at one end with 0 , indicating no pain, and 10 at the other, indicating the worst pain imaginable. The participant placed a mark along the line corresponding to the intensity of their pain. This scale has been demonstrated to be a reliable and valid measure of pain intensity, and it is sensitive to clinical changes in pain [26,27]. Changes of $1.1-1.2 \mathrm{~cm}$ indicate a minimal clinical improvement [28].

The Spanish version of the PCS was used to assess the degree of pain catastrophizing [21]. The PCS has 13 items 

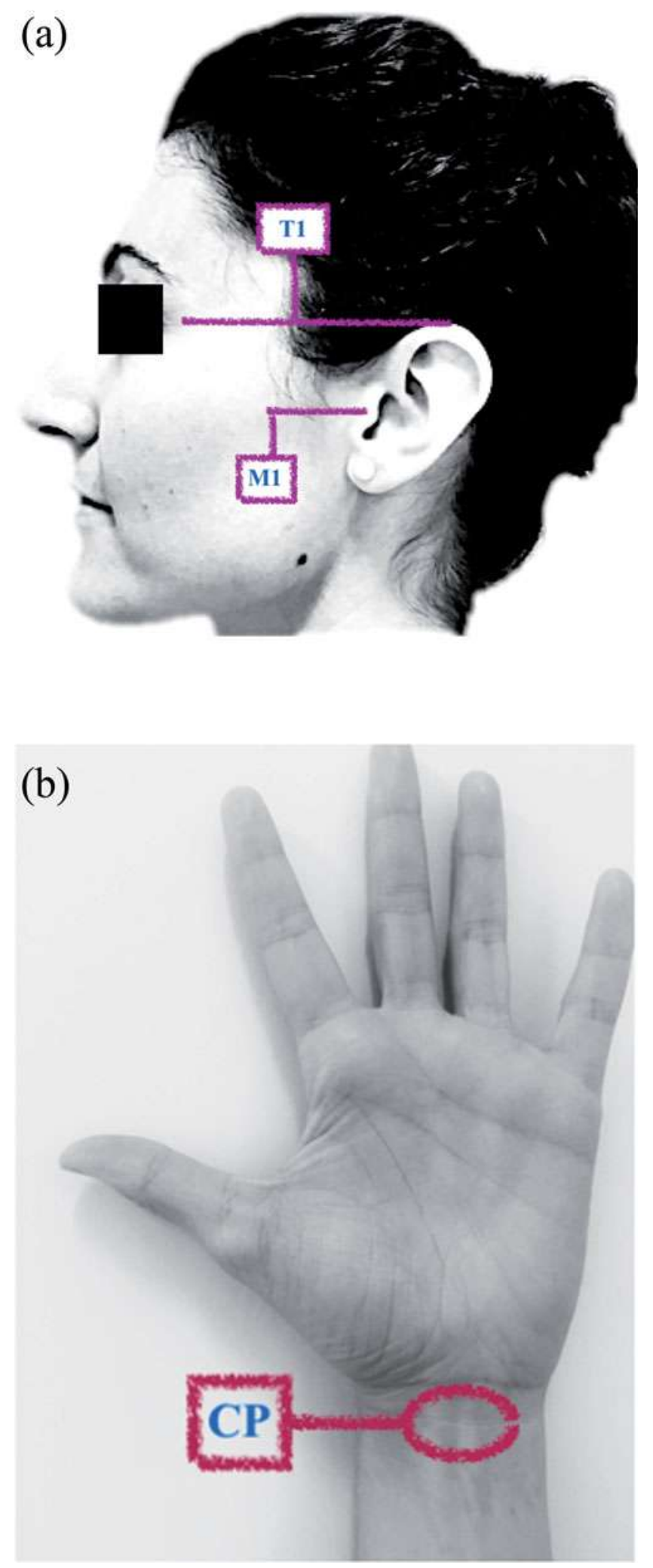

Figure 1. Pressure pain threshold measurement sites at trigeminal and extratrigeminal regions.

that comprise three dimensions: rumination, magnification and helplessness. Each item is scored from 0 (not at all) to 4 (always), and scores range from 0 to 52, with higher scores indicating a greater degree of catastrophizing. This scale has demonstrated acceptable psychometric properties and internal consistency is 0.79 [21]. The minimal detectable change is 10.45 [29].

The CF-PDI was used to assess pain, disability and functional status of the mandibular and craniofacial regions [22]. This self-administered questionnaire consists of 21 items, with a score ranging from 0 to 63 . The CF-PDI is an objective tool for assessing pain and disability in craniofacial pain patients [22]. The CF-PDI shows good psychometric properties and the minimal detectable change is 7 points [22].

The Spanish version of the HIT-6 was used to assess the impact and severity of headache on the patient's life [23]. This questionnaire consists of six items and has demonstrated acceptable psychometric properties and has been validated for patients with CM [30]. The results are stratified into four grades of impact: little or no impact (HIT-6 score: 36-49), moderate impact (HIT-6 score: 50-55), important impact (HIT-6 score: 56-59), and severe impact (HIT-6 score: 60-78). The minimally important difference of the HIT-6 in patients with chronic daily headache has been estimated to be between 2.3 and 2.7 [31].

The Spanish version of the HADS was used to assess the presence of depression and anxiety in the patients [24]. This scale includes 14 items, which are rated on a four-point Likert-type scale. Two subscales assessed depression and anxiety independently. The internal consistency is 0.90 for full scale; 0.84 for the depression subscale and 0.85 for the anxiety subscale [24]. The minimal important difference of the HADS is around 1.5 [32].

\section{Outcome measures}

An analog algometer was used to assess the PPT (Wagner Instruments, Greenwich, CT). This instrument consists of a $1 \mathrm{~cm}$ diameter hard rubber tip attached to the plunger of a pressure (force) gauge. The dial of the gauge is calibrated in $\mathrm{kg} / \mathrm{cm}^{2}$, and the range of the algometer is 0.0 to $10.0 \mathrm{~kg}$ with $0.1 \mathrm{~kg}$ divisions. Previous research has shown that the reliability of pressure algometry is as high as intra-class correlation coefficient (ICC), ICC $=0.91$ ( $95 \%$ confidence interval, Cl: 0.82-0.97) [33].

Before the evaluation, two specific cutaneous regions around the temporalis and masseter were marked with a pencil. The PPT was measured at two trigeminal sites. One was applied to the skin overlying the anterior fibers of the temporalis muscle (T1), $3 \mathrm{~cm}$ above the line between the lateral edge of the eye and the anterior part of the helix and other were applied to the skin overlying the masseter muscle, $2.5 \mathrm{~cm}$ anterior to the tragus and $1.5 \mathrm{~cm}$ inferior to the zygomatic arch (M1), based on a previously published protocol [25] (Figure 1). The extra-trigeminal point (CP) was established in the palmar region of the wrist, in the middle point of the distal part between the ulnar and the radius (Figure 1). The algometer was held perpendicular to the skin, and the participants were told to immediately alert the assessor when the pressure turned into pain; the pressure at that point was recorded [25].

Measurements were made when the participant was seated with his/her back supported, with feet resting on the 


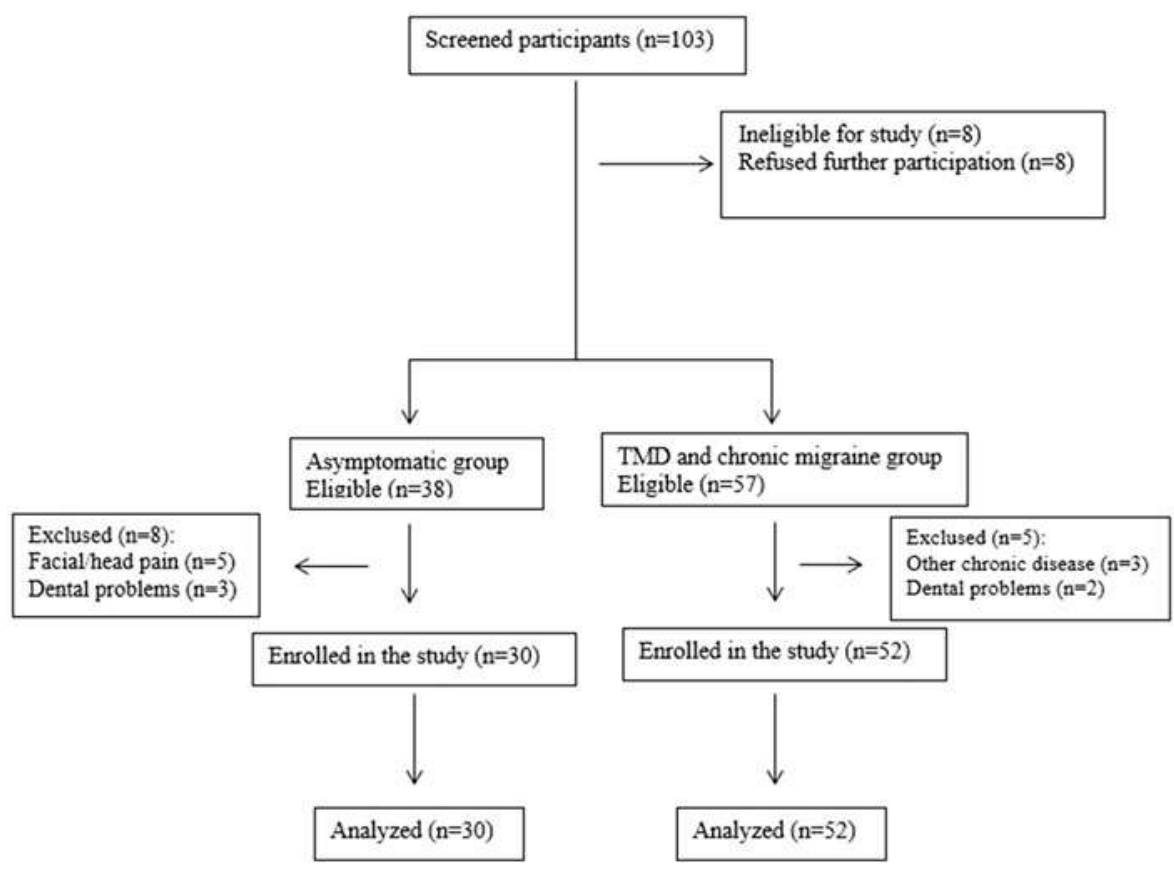

Figure 2. Flowchart of sample selection.

floor, in a comfortable upright position. The measurements were taken by the same blinded evaluator who had more than 5 years of clinical experience in craniofacial pain. Three measurements were taken for each point, bilaterally, with an interval of 30 seconds between measurements. Bilateral data for each point were analyzed, and no significant differences were found. The data of PPTs used for analysis corresponded to the mean of the three measures for each point and the mean of both sides of the participant [25].

The MMO without pain was registered using a digital pachymeter placed between the edges of the maxillary and mandibular incisors [34]. Participants were given the following instructions: 'open your mouth as wide as possible without causing pain or discomfort' $[25,34]$. The vertical range of motion corresponds to the last measurement of three opening movements made by the patient [34].

\section{Sample size}

The sample size was estimated with G*Power version 3.1.7 for Windows (G*Power $(C)$ from the University of Dusseldorf, Dusseldorf, Germany). The power calculation was set to detect inter-group differences in the PPT (trigeminal region). A Student's t-test analysis for independent samples was used to detect the mean difference between groups, as this was the main variable of interest. An effect size of 0.65 (medium) was used, based on a pilot study with a sample of 14 subjects ( 7 from the patient group and 7 from the control group), where the statistical power was set at $80 \%$ (1- $\beta$ error probability) and the $\alpha$ error level probability was set at 0.05 . The recommended sample size was 60 participants (30 per group). Given that the secondary aim was to assess the association between variables through a regression analysis with five predictors, it was necessary to expand the sample in CM and TMD group. For the regression analysis, the rule of 10 cases per variable was applied to obtain reasonably stable estimates of the regression coefficients [35]. Thus, it was estimated that a total of 50 participants in CM and TMD group would be required.

\section{Data analysis}

Descriptive statistics were generated for the sociodemographic, psychological and pain-related variables and sensorimotor measures. Continuous variables are presented as the mean$\mathrm{s} \pm$ standard deviation (SD) and ranges, and categorical variables are presented as absolute numbers and relative frequency (percentage). A chi-square test was used to compare categorical variables. The Kolmogorov-Smirnov test was used to determine whether the variables followed a normal distribution.

A Student's $t$-test for independent samples was used to compare the outcomes between the two groups. Effect sizes (Cohen's $d$ ) were calculated for the outcome variables. According to Cohen's method, the magnitude of the effect was classified as small $(0.20-0.49)$, medium (0.50-0.79), or large (0.8) [36].

The relationship between sensorimotor measures (PPT and $\mathrm{MMO}$ ) and self-reported pain-related and psychological measures was examined using Pearson correlation coefficients. A Pearson correlation coefficient greater than 0.60 was taken to indicate a strong correlation, a coefficient between 0.30 and 0.60 was taken to indicate a moderate correlation, and a coefficient below 0.30 was taken to indicate a weak or very weak correlation [37].

A stepwise multiple linear regression analysis was performed to estimate the strength of the associations between the results obtained for M1 (point trigeminal region) [model 1], T1 (point trigeminal region) [model 2], PC (point extra-trigeminal region) [model 3], and MMO [model 4] (criterion variables). Psychological and pain-related variables were used as predictor 
Table 1. General data for the subjects analyzed.

\begin{tabular}{lccc} 
Demographic and clinical data & $\begin{array}{c}\text { CM and TMD } \\
(n=52)\end{array}$ & $\begin{array}{c}\text { CG } \\
(n=30)\end{array}$ & $p$ value \\
\hline Age, mean \pm SD $(\mathrm{y})$ & $46.2 \pm 9.5$ & $47.4 \pm 10$ & .59 \\
Gender, $n(\%)^{+}$ & & & .16 \\
$\quad$ Female & $48(92.3)$ & $24(80)$ & \\
$\quad$ Male & $4(7.7)$ & $6(20)$ & .58 \\
Weight, mean \pm SD (kg) & $66.9 \pm 11.6$ & $68.3 \pm 9.7$ & .19 \\
Height, mean \pm SD $(\mathrm{cm})$ & $164.6 \pm 6.8$ & $168.3 \pm 7$ & .45 \\
Body Mass Index, mean \pm SD $\left(\mathrm{Kg} / \mathrm{m}^{2}\right)$ & $24.7 \pm 4.1$ & $24 \pm 2.7$ & - \\
Duration of pain, mean $\pm S D(\mathrm{y})$ & $25.1 \pm 10.5$ & - & - \\
VAS, mean $\pm S D(\mathrm{~cm})$ & $7.3 \pm 1.3$ & - & -
\end{tabular}

Abbreviations: CG: Control Group; CM: Chronic Migraine; SD: Standard Deviation; TMD: Temporomandibular Disorders; VAS: Visual Analogue Scale; Y: years.

All comparisons were performed using the Student unpaired $t$ test. When another test was used it is highlighted: ${ }^{+}$chi-square test.

Table 2. Descriptive statistics for psychological, disability and sensorimotor variables.

\begin{tabular}{lccccc}
\hline Variables & CM and TMD $(n=52)$ & CG $(n=30)$ & & & \\
Mean \pm SD & Mean \pm SD & Mean differences & $95 \%$ Cl (lower; higher) & Effect size $(d)$ \\
\hline HIT-6 & $66.25 \pm 5.55$ & $38.43 \pm 4.05$ & -27.82 & $(-30.12 ;-25.51)^{* *}$ & 5.5 \\
PCS & $28.79 \pm 11.25$ & $4.83 \pm 9.89$ & -23.95 & $(-28.87 ;-19.04)^{* *}$ & 2.22 \\
CF-PDI & $22.85 \pm 12.13$ & $1.83 \pm 1.51$ & -21.13 & $(-25.45 ;-16.57)^{* *}$ & 2.16 \\
HADS depression & $6.27 \pm 5.33$ & $2.80 \pm 3.16$ & -3.47 & $(-5.59 ;-1.34)^{* *}$ & 0.74 \\
HADS anxiety & $8.29 \pm 4.85$ & $4.13 \pm 3.08$ & -4.15 & $(-6.11 ;-2.19)^{* *}$ & 0.97 \\
PPT (kg/cm $\left.{ }^{2}\right)$ & & & & & 1.04 \\
T1 & $1.95 \pm 0.52$ & $2.44 \pm 0.37$ & 0.49 & $(0.27 ; 0.70)^{* *}$ & 1.06 \\
M1 & $1.83 \pm 0.46$ & $2.31 \pm 0.44$ & 0.47 & $(0.27 ; 0.68)^{* *}$ & 0.6 \\
CP & $3.30 \pm 0.84$ & $3.74 \pm 0.50$ & 0.44 & $(0.15 ; 0.74)^{* *}$ & 1.41 \\
Pain-free MMO (mm) & $32.17 \pm 8.85$ & $43.63 \pm 6.75$ & 11.46 & $(7.74 ; 15.18)^{* *}$ & \\
\hline Abbreviation: CF-PD: Crani & & &
\end{tabular}

Abbreviations: CF-PDI: Craniofacial and Disability Inventory; CG: Control Group; CM: Chronic Migraine; CP: Control Point; HADS: Hospital Anxiety and Depression scale; HIT-6: Headache Impact Test; M1: masseter muscle; MMO: Maximal Mouth Opening; PCS: Pain Catastrophizing Scale; PPT: Pressure Pain Threshold; SD: Standard Deviation; T1: temporal muscle; TMD: Temporomandibular Disorders.

${ }^{* *} p<.01 ;{ }^{*} p<.05$.

Table 3. Pearson's correlation coefficients of psychological, disability and sensorimotor variables.

\begin{tabular}{lllll}
\hline $\begin{array}{l}\text { Psychological-disability/Sensorimotor } \\
\text { variables }(n=52)\end{array}$ & PPT T1 & PPT M1 & PPT CP & Pain-free MMO \\
\hline HIT-6 & $-0.383^{* *}$ & $-0.407^{* *}$ & -0.258 & $-0.410^{* *}$ \\
PCS & $-0.342^{*}$ & $-0.278^{*}$ & -0.242 & -0.144 \\
CF-PDI & $-0.461^{* *}$ & $-0.283^{*}$ & -0.140 & $-0.507^{* *}$ \\
HADS depression & $-0.372^{* *}$ & $-0.438^{* *}$ & $-0.349^{*}$ & $-0.346^{*}$ \\
HADS anxiety & $-0.288^{*}$ & $-0.282^{*}$ & $-0.324^{*}$ & -0.173 \\
\hline
\end{tabular}

Abbreviations: CF-PDI: Craniofacial and Disability Inventory; CP: Control Point; HADS: Hospital Anxiety and Depression scale; HIT-6: Headache Impact Test; M1: masseter muscle; MMO: Maximal Mouth Opening; PCS: Pain Catastrophizing Scale; PPT: Pressure Pain Threshold; T1: temporal muscle.

$*^{*} p<.01 ; * p<.05$.

variables. Variance inflation factors (VIFs) were calculated to determine if multi-collinearity existed in any of the three models. The strength of the associations was examined using regression coefficients $(\beta), p$ values, and adjusted $R^{2}$ values. Standardized beta coefficients were reported for each predictor variable included in the final reduced models to allow for direct comparisons between the predictor variables in the regression model and the criterion variable being studied. All data analyses were performed using SPSS for Windows version 21.0 (SPSS Inc., Chicago, IL), where $\alpha$ was set to 0.05 .

\section{Results}

A total of 95 participants were included in the study and assigned to one of two groups: 38 participants to the asymptomatic group, and 57 to the TMD and CM group. In the asymptomatic group, eight participants were excluded due to a history of facial/head pain and dental problems. In the TMD and CM group, five participants were excluded due to the presence of other chronic disease and dental problems. Finally, 82 participants were included for the final analysis. Figure 2 shows the selection and progression of participants throughout the study.

Table 1 shows that no statistically significant differences existed between the two groups with regard to any sociodemographic variables. Table 2 shows statistically significant differences between the two groups with regard to psychological and physical variables. The present study found that the HIT-6, PCS and CF-PDI questionnaires each showed a difference of more 
Table 4. Multiple linear regression analysis of psychological, disability and sensorimotor variables. Patients with CM and TMD $(n=52)$.

\begin{tabular}{|c|c|c|c|c|c|c|}
\hline Criterion variable & Predictor variables & Regression coefficient $(B)$ & Standardized coefficient $(\beta)$ & Significance $(p)$ & VIF & Adjusted $R^{2}$ \\
\hline \multirow[t]{6}{*}{ PPT M1 } & $\begin{array}{l}\text { HADS Depression } \\
\text { Excluded variables }\end{array}$ & -0.038 & -0.438 & .001 & 1.000 & 0.18 \\
\hline & PCS & - & -0.042 & .79 & 1.484 & - \\
\hline & CF-PDI & - & -0.12 & .397 & 1.215 & - \\
\hline & HADS Anxiety & - & 0.059 & .75 & 2.024 & - \\
\hline & HIT-6 & - & -0.259 & .071 & 1.275 & - \\
\hline & Pain-free MMO & - & 0.157 & .249 & 1.136 & - \\
\hline \multirow[t]{6}{*}{ PPT CP } & $\begin{array}{l}\text { HADS Depression } \\
\text { Excluded variables }\end{array}$ & -0.055 & -0.349 & .011 & 1.000 & 0.10 \\
\hline & PCS & - & -0.063 & .701 & 1.484 & - \\
\hline & CF-PDI & - & 0.008 & .957 & 1.215 & - \\
\hline & HADS Anxiety & - & -0.153 & .422 & 2.024 & - \\
\hline & HIT-6 & - & -0.122 & .421 & 1.275 & - \\
\hline & Pain-free MMO & - & -0.092 & .518 & 1.136 & - \\
\hline \multirow[t]{6}{*}{ PPT T1 } & $\begin{array}{l}\text { CF-PDI } \\
\text { Excluded variables }\end{array}$ & -0.02 & -0.461 & .001 & 1.000 & 0.20 \\
\hline & PCS & - & -0.138 & .353 & 1.376 & - \\
\hline & HADS Depression & - & -0.216 & .12 & 1.215 & - \\
\hline & HADS Anxiety & - & -0.121 & .385 & 1.196 & - \\
\hline & HIT-6 & - & -0.238 & .082 & 1.186 & - \\
\hline & Pain-free MMO & - & 0.122 & .408 & 1.346 & - \\
\hline \multirow[t]{5}{*}{ Pain-free MMO } & $\begin{array}{l}\text { CF-PDI } \\
\text { Excluded variables }\end{array}$ & -0.37 & -0.507 & $<.001$ & 1.000 & 0.24 \\
\hline & PCS & - & 0.167 & .247 & 1.376 & - \\
\hline & HADS Depression & - & -0.162 & .231 & 1.215 & - \\
\hline & HADS Anxiety & - & 0.038 & .778 & 1.196 & - \\
\hline & HIT-6 & - & -0.248 & .061 & 1.186 & - \\
\hline
\end{tabular}

Abbreviations: CF-PDI: Craniofacial and Disability Inventory; CM: Chronic Migraine; CP: Control Point; HADS: Hospital Anxiety and Depression scale; HIT-6: Headache Impact Test; M1: masseter muscle; MMO: Maximal Mouth Opening; PCS: Pain Catastrophizing Scale; PPT: Pressure Pain Threshold; T1: temporal muscle; TMD: Temporomandibular Disorders.

than 20 points between the groups and the PPT exhibited a difference greater than $0.4 \mathrm{~kg} / \mathrm{cm}^{2}$ between the groups.

\section{Correlations analysis}

The Pearson's correlation coefficients obtained in the present analysis of the bivariate relationships among the self-questionnaire for pain-related and psychological variables and sensorimotor variables (e.g. PPT, pain-free MMO) are given in Table 3. A relationship was found between the psychological variables and the PPT T1 and M1 values. The PPT CP value was found to be related to the HADS Depression and HADS Anxiety scales. Finally, the pain-free MMO was found to exhibit a moderate relationship with CF-PDI $(r=-0.507$; $p<.01)$ and HIT-6 $(r=-0.410 ; p<.01)$ and a weaker association with the HADS Depression scale $(r=-0.346 ; p<.05)$. PPT T1 values showed an association with CF-PDI $(r=-0.461$; $p<.01)$, and PPT M1 values showed an association with the HADS Depression scale $(r=-0.438 ; p<.01)$. All correlations were negative.

\section{Multiple linear regression analysis}

A linear regression analysis was performed to evaluate the contributors to PPT M1, PPT CP, PPT T1 and pain-free MMO, taking into account all of the self-reported results for painrelated and psychological measures and the sensorimotor variables measured in the patient group with CM and TMD. The results are presented in Table 4. The criterion variable PPT M1 was predicted by the HADS Depression scale $(p=.001$; explaining $18 \%$ of the variance). This variable was not significant for the rest of the variables included in the model. PPT T1 was predicted by CF-PDI ( $p=.001 ; 20 \%$ of the variance); none of the other variables were significant predictors. PPT CP was predicted by the HADS Depression scale $(p=.011 ; 10 \%$ of the variance), and none of the other variables were significant predictors. Finally, pain-free MMO was predicted by CF-PDI ( $p=<.001 ; 24 \%$ of the variance), and the remaining variables were not significant.

\section{Discussion}

The results confirm the hypothesis of this study and show that generalized hyperalgesia is present in the trigeminal and extra-trigeminal regions of patients with $C M$ and TMD in comparison to healthy subjects. It should also be noted that the PPT for the trigeminal and extra-trigeminal regions was predicted by depressive symptoms, disability and craniofacial and craniomandibular pain; according to the multiple linear regression analysis, this latter variable was also predictive of MMO.

It has been proposed that the pathophysiology of migraine may be associated with a central sensitization process $[5,6]$. Neurophysiologically, the reception of nociceptive afferents in the central nervous system is part of central sensitization, which is a key process in the onset and maintenance of chronic pain. In patients with migraine, this process takes place in the caudate nucleus of the trigeminal, where the extra-cranial trigeminal nociceptive inputs derived from craniofacial structures also converge $[9,13]$. Migraine-related allodynia develops at the site where headache occurs, normally in the ophthalmic region of the trigeminal nerve, and propagates throughout the face, scalp, body, and upper and lower limbs [6]. There is now evidence that patients with 
TMD present with generalized hyperalgesia [16]. This finding supports the view that central sensitization mechanisms govern both conditions. These theories could explain the findings obtained in the current study concerning the PPT values in the trigeminal region, and these findings are consistent with those of numerous other studies [38,39]. Pinto reported a lower PPT in patients with migraine and myofascial pain in the masticatory muscles in comparison to asymptomatic subjects, which is consistent with the findings obtained in this study [40]. Some authors have reported a decrease in PPT in both the trigeminal and extra-trigeminal regions in patients with migraine [41], as well as in patients with TMD, in comparison to a control group of asymptomatic subjects [42].

In the current study found various associations between the physical, psychological and disability variables observed in group of the patients. Thus, the PPT values recorded in the trigeminal regions above two points for the masticatory muscles (PPT T1 and PPT M1) are negatively correlated with the HIT-6, PCS, CF-PDI and HADS scores. A previous study demonstrated a strong positive correlation between sensitivity and headache frequency [43]. Other study showed that patients with TMD suffer greater levels of stress, depression and anxiety than asymptomatic subjects [3], and exhibit higher levels of pain-related catastrophization [2]. The predominant psychological aspects of patients with $\mathrm{CM}$ are anxiety and depression [10]. CM and TMD have a negative effect on the emotional health of individuals. Similarly, psychological and emotional alterations often coexist with chronic pain, and these factors maintain and worsen pain symptoms [44]. In this regard, it should be noted that the results from the present study show that depressive symptoms are predictors for some of the points at which the PPT was measured in migraine patients (i.e. M1 and CP). It is important to note that psychological factors may be responsible for increasing the severity and intensity of headaches [10]. A possible explanation for the correlations that the authors found could be the central sensitization process.

Migraine pain manifests in the ophthalmic branch (V1) of the trigeminal nerve, whereas TMD pain manifests in the upper (V2) and lower jaws (V3). Migraine patients often present with pain in the TMJ region and neighboring structures in addition to the characteristic headaches [45]. This observation highlights the importance of including the orofacial region as part of assessment and treatment in patients with $\mathrm{CM}$. The current study found a negative correlation between pain-free MMO and HIT-6, CF-PDI and, to a lesser extent, depression scores. Several previous studies have found an association between TMD and headache [8,11]. Exist one type of headache attributed to TMD is listed as a secondary headache in the ICHD-III [18], unlike the patients in this study who present $\mathrm{CM}$, one type of primary headache, diagnosed by a specialist headache neurologist and diagnosis of myofascial TMD. One diagnostic criteria for headache attributed to TMD is a limited range of movement in the lower jaw [18]. As such, the authors should consider the possibility that some patients with CM may present with comorbid TMDrelated headache. However, it should also be noted that other patients with various types of craniofacial pain (e.g. trigeminal neuralgia) also present with pain and limited function and range of movement of the lower jaw [46]. Another study performed in patients with fibromyalgia who also presented with TMD found reduced mouth opening movement in comparison to a group of healthy subjects [47]. The present study found that craniofacial and craniomandibular pain and disability predicted $\mathrm{MMO}$, with an explained variance of $24 \%$. The authors believe that the possible limitation in MMO occurs due to the onset of TMD rather than migraine, although future studies should clarify this supposition.

The results demonstrate the existence of an association between mandibular movement, craniofacial sensitivity, and psychosocial and disability factors in patients with CM and TMD. As such, the craniofacial region must be evaluated, and a multidisciplinary treatment model must be established by incorporating biopsychosocial perspectives when treating chronic pain.

The current study has several limitations. First, $87.8 \%$ of the sample was female, potentially limiting the study's generalizability. However, scientific evidence suggests a high prevalence of migraine and craniofacial pain in females $[1,7,8]$. Second, in this study, the authors did not report the menstrual status and oral contraceptive use of the female participants. Another important limitation was that medication was not recorded or analyzed in this study; it is important that this might be addressed in future studies.

This study has a cross-sectional design and longitudinal studies are necessary to establish causal relationships. Finally, this study compared different variables for patients with $\mathrm{CM}$ and TMD and asymptomatic participants; thus, no comparison with CM patients without TMD or patients who presented with TMD alone was made. Future studies should include these two groups to establish a more accurate and specific conclusion.

This study concluded that patients with CM and TMD are more likely to present with generalized mechanical hyperalgesia in the trigeminal and extra-trigeminal regions than their healthy counterparts. Moreover, this study found an association between sensorimotor, psychological and disability variables, and also be noted that the sensorimotor variables were predicted by psychological and disability variables.

\section{Disclosure statement}

No potential conflict of interest was reported by the authors.

\section{References}

[1] Lipton RB, Bigal ME, Diamond M, et al. Migraine prevalence, disease burden, and the need for preventive therapy. Neurology. 2007;68:343-349.

[2] Costa YM, Porporatti AL, Stuginski-Barbosa J, et al. Additional effect of occlusal splints on the improvement of psychological aspects in temporomandibular disorder subjects: a randomized controlled trial. Arch Oral Biol. 2015;60:738-744.

[3] Giannakopoulos NN, Keller L, Rammelsberg P, et al. Anxiety and depression in patients with chronic temporomandibular pain and in controls. J Dent. 2010;38:369-376.

[4] Bevilaqua-Grossi D, Lipton RB, Napchan U, et al. Temporomandibular disorders and cutaneous allodynia are associated in individuals with migraine. Cephalalgia. 2010;30:425-432. 
[5] Merskey $\mathrm{H}$. Terms and taxonomy: paper tools at the cutting edge of study. In: Merskey H, Loeser JD, Dubner R, editors. The paths of pain 1975-2005. Seattle: IASP Press; 2005. p. 329-337.

[6] Treede RD, Handwerker HO, Baumgartner U, et al. Hyperalgesia and allodynia: taxonomy, assessment, and mechanisms. In: Brune $\mathrm{K}$, Handwerker $\mathrm{HO}$, editors. Hyperalgesia: molecular mechanisms and clinical implications. Seattle: IASP Press; 2004. p. 1-15.

[7] Blumenfeld AM, Varon SF, Wilcox TK, et al. Disability, HRQoL and resource use among chronic and episodic migraineurs: results from the International Burden of Migraine Study (IBMS). Cephalalgia. 2011;31:301-315.

[8] Gonçalves DAG, Bigal ME, Jales LCF, et al. Headache and symptoms of temporomandibular disorder: an epidemiological study. Headache. 2010;50:231-241.

[9] Speciali JG, Dach F. Temporomandibular dysfunction and headache disorder. Headache. 2015;55:72-83.

[10] Tarantino S, De Ranieri C, Dionisi C, et al. Clinical features, anger management and anxiety: a possible correlation in migraine children. J Headache Pain. 2013;14:39-46.

[11] Franco AL, Gonçalves DAG, Castanharo SM, et al. Migraine is the most prevalent primary headache in individuals with temporomandibular disorders. J Orofac Pain. 2010;24:287-292.

[12] Gonçalves DAG, Camparis CM, Speciali JG, et al. Temporomandibular disorders are differentially associated with headache diagnoses a controlled study. Clin J Pain. 2011;27:611-615.

[13] Fernandes G, Franco AL, Gonçalves DA, et al. Temporomandibular disorders, sleep bruxism, and primary headaches are mutually associated. J OrofacPain. 2013;27:14-20.

[14] Filatova E, Latysheva N, Kurenkov A, Evidence of persistent central sensitization in chronic headaches: a multi-method study. J Headache Pain. 2008;9:295-300.

[15] Stuginski-Barbosa J, Santos Silva R, Ortigosa Cunha C, et al. Pressure pain threshold and pain perception in temporomandibular disorder patients: is there any correlation?. Rev Dor. 2015;16:22-26.

[16] Fernandez-de-las-Peñas C, Galan-del-Rio F, Ortega-Santiago R, et al. Bilateral thermal hyperalgesia in trigeminal and extra-trigeminal regions in patients with myofascial temporomandibular disorders. Exp Brain Res. 2010;202:171-179.

[17] Vandenbroucke JP, von Elm E, Altman DG, et al. Strengthening the Reporting of Observational Studies in Epidemiology (STROBE), Epidemiology. 2007;18:805-835.

[18] Headache Classification Committee of the International Headache S. The international classification of headache disorders, 3rd edition (beta version). Cephalalgia. 2013;33:629-808.

[19] Dworkin SF, Leresche L. Research diagnostic criteria for temporomandibular disorders: review, criteria, examinations and specifications, critique. J Craniomandib Disord. 1992;6:301-355.

[20] Huskisson EC, Measurement of pain. Lancet. 1974;2:1127-1131.

[21] Garcia-Campayo J, Rodero B, Alda MS, et al. Validation of the Spanish version of the Pain Catastrophizing Scale in fibromyalgia. Med Clin-Barcelona. 2008;131:487-492.

[22] La Touche R, Pardo-Montero J, Gil-Martinez A, et al. Craniofacial pain and disability inventory (CF-PDI): development and psychometric validation of a new questionnaire. Pain Physician. 2014;17: 95-108.

[23] Gandek B, Alacoque J, Uzun V, et al. Translating the short-form headache impact test (HIT-6) in 27 countries: methodological and conceptual issues. Qual Life Res. 2003;12:975-979.

[24] Herrero MJ, Blanch J, Peri JM, et al. A validation study of the hospital anxiety and depression scale (HADS) in a Spanish population. Gen Hosp Psychiatry. 2003;25:277-283.

[25] La Touche R, Paris-Alemany A, Von Piekartz $\mathrm{H}$, et al. The influence of cranio-cervical posture on maximal mouth opening and pressure pain threshold in patients with myofascial temporomandibular pain disorders. Clin J Pain. 2011;27:48-55.

[26] Bijur PE, Silver W, Gallagher EJ. Reliability of the visual analog scale for measurement of acute pain. Acad Emergency Med. 2001;8:1153-1157.
[27] Jensen MP, Turner JA, Romano JM, et al. Comparative reliability and validity of chronic pain intensity measures. Pain. 1999;83: 157-162.

[28] Emshoff R, Bertram S, Emshoff I. Clinically important difference thresholds of the visual analog scale: a conceptual model for identifying meaningful intraindividual changes for pain intensity. Pain. 2011;152:2277-2282.

[29] Monticone $M$, Baiardi $P$, Ferrari $S$, et al. Development of the Italian version of the Pain Catastrophising Scale (PCS-I): cross-cultural adaptation, factor analysis, reliability, validity and sensitivity to change. Qual Life Res. 2012;21:1045-1050.

[30] Rendas-Baum $R$, Yang $M$, Varon SF, et al. Validation of the Headache Impact Test (HIT-6) in patients with chronic migraine. Health Qual Life Outcomes. 2014;12:117-126.

[31] Coeytaux RR, Kaufman JS, Chao R, et al. Four methods of estimating the minimal important difference score were compared to establish a clinically significant change in Headache Impact Test. J Clin Epidemiol. 2006;59:374-380.

[32] Puhan MA, Frey $M$, Büchi $S$, et al. The minimal important difference of the hospital anxiety and depression scale in patients with chronic obstructive pulmonary disease. Health Qual Life Outcomes. 2008;6:46.

[33] Chesterton LS, Sim J, Wright CC, et al. Interrater reliability of algometry in measuring pressure pain thresholds in healthy humans, using multiple raters. Clin J Pain. 2007;23:760-766.

[34] Gonçalves DAG, Camparis CM, Speciali JG, et al. Treatment of comorbid migraine and temporomandibular disorders: a factorial, double-blind, randomized, placebo-controlled study. J Orofac Pain. 2013;27:325-335.

[35] Peduzzi P, Concato J, Kemper E, et al. A simulation study of the number of events per variable in logistic regression analysis. J Clin Epidemiol. 1996;49:1373-1379.

[36] Cohen J. Statistical power analysis for the behavioral-sciences. 2nd ed. Hillsdale, New Jersey: Lawrence Earlbaum Associates; 1988, pp.67-107.

[37] Hinkle D, Jurs S, Wiersma W, editors. Applied statistics for the behavioral sciences. Boston: Houghton-Mifflin; 1998.

[38] Grossi DB, Chaves TC, Gonçalves MC, et al. Pressure pain threshold in the craniocervical muscles of women with episodic and chronic migraine: a controlled study. Arq Neuropsiquiatr. 2011;69: 607-612.

[39] Florencio LL, Giantomassi MC, Carvalho GF, et al. Generalized pressure pain hypersensitivity in the cervical muscles in women with migraine. Pain Med. 2015;16:1629-1634.

[40] Sales Pinto LM, de Carvalho JJ, Cunha CO, et al. Influence of myofascial pain on the pressure pain threshold of masticatory muscles in women with migraine. Clin J Pain. 2013;29:362-365.

[41] Grosu O, Moldovanu I, Diaconu N. Cranial and extracranial pain pressure thresholds evaluated in chronic and episodic migraine. Neurol. 2012;259:S143-S144.

[42] Bragatto MM, Bevilaqua-Grossi D, Regalo SC, et al. Associations among temporomandibular disorders, chronic neck pain and neck pain disability in computer office workers: a pilot study. J Oral Rehabil. 2016;43:321-332.

[43] Jensen R, Rasmussen BK, Pedersen B, et al. Muscle tenderness and pressure pain thresholds in headache. A population study. Pain. 1993;52:193-199.

[44] Campi LB, Camparis CM, Jordani PC, et al. Influence of biopsychosocial approaches and self-care to control chronic pain and temporomandibular disorders. Rev Dor. 2013;14:219-222.

[45] Gonçalves DA, Camparis CM, Franco AL, et al. How to investigate and treat: migraine in patients with temporomandibular disorders. Curr Pain Headache Rep. 2012;16:359-364.

[46] Ichida MC, da Silva LA, Teixeira MJ, et al. Functional and sensory evaluation of patients with idiopathic trigeminal neuralgia: comparison with controls. Clin Neurol Neurosur. 2015;130:114-121.

[47] Garcia-Moya E-J, Montiel-Company J-M, Almerich-Silla J-M. Casecontrol study of craniomandibular disorders in patients with fibromyalgia. J Clin Exp Dent. 2015;7:293-298. 